\title{
Correction to: Cobalt(II) Complexes as Single-Ion Magnets
}

Shalini Tripathi, Atanu Dey, Maheswaran Shanmugam, Ramakirushnan Suriya Narayanan, and Vadapalli Chandrasekhar

\section{Correction to:}

Chapter "Cobalt(II) Complexes as Single-Ion Magnets" in:

S. Tripathi et al., Topics in Organometallic Chemistry, DOI: $10.1007 / 3418 \_2018 \_8$

In the Chapter opener page, the spelling of the author is inadvertently misspelt as Vadapalli Chandrsekhar, which is now corrected as Vadapalli Chandrasekhar. 